\title{
Music in the exercise domain: a review and synthesis (Part II)
}

\author{
Costas I. Karageorghis* and David-Lee Priest \\ School of Sport and Education, Brunel University, London, UK \\ (Received 8 June 2011; final version received 6 October 2011)
}

Since a 1997 review by Karageorghis and Terry, which highlighted the state of knowledge and methodological weaknesses, the number of studies investigating musical reactivity in relation to exercise has swelled considerably. In this two-part review paper, the development of conceptual approaches and mechanisms underlying the effects of music are explicated (Part I), followed by a critical review and synthesis of empirical work (spread over Parts I and II). Pre-task music has been shown to optimise arousal, facilitate task-relevant imagery and improve performance in simple motoric tasks. During repetitive, endurance-type activities, self-selected, motivational and stimulative music has been shown to enhance affect, reduce ratings of perceived exertion, improve energy efficiency and lead to increased work output. There is evidence to suggest that carefully selected music can promote ergogenic and psychological benefits during highintensity exercise, although it appears to be ineffective in reducing perceptions of exertion beyond the anaerobic threshold. The effects of music appear to be at their most potent when it is used to accompany self-paced exercise or in externally valid conditions. When selected according to its motivational qualities, the positive impact of music on both psychological state and performance is magnified. Guidelines are provided for future research and exercise practitioners.

Keywords: pre-task music; asynchronous music; synchronous music; post-task music

In Part I of this two-part article we gave a broad introduction to the area of music in exercise detailing how the number of published studies has proliferated in recent years $(k=62)$ since the 1997 review of Karageorghis and Terry $(k=13)$. We then critically appraised the theoretical approaches used to underpin work in this field and explained the mechanisms used by researchers to explain the effects of music on exercisers. Thereafter we presented a review and synthesis of empirical work that had investigated pre-task music and the in-task asynchronous use of music. In Part II we present a review and synthesis of empirical work that has examined the in-task synchronous use of music, the longitudinal effects of music and post-task music. The synchronous application of music entails an exerciser's conscious coordination between their movements and the tempo or rhythmical qualities of music. The longitudinal effects section addresses the few studies that have investigated the repeated use of a music programme, while the post-task section reflects an entirely new area of research examining the recuperative effects of music in exercise (see Terry \& Karageorghis, 2011). A general discussion pertaining to Part I and Part II of this

*Corresponding author. Email: costas.karageorghis@brunel.ac.uk 
article follows, along with suggestions for future research and implications for exercise practitioners.

\section{In-task synchronous use of music}

Music is used synchronously to accompany repetitive endurance tasks such as cycle ergometry, walking and running (e.g., Karageorghis et al., 2009; Simpson \& Karageorghis, 2006). The validity of several early studies was compromised by tasks that were difficult or impossible to standardise (see Karageorghis \& Terry, 1997). The psychophysical benefits of synchronous music use are equivalent to those observed in the asynchronous use of music, while the ergogenic effects consistently exceed those found in its asynchronous application.

Schneider, Askew, Abel, and Strüder (2010) proposed that human movement and rhythmical perception are both bound to the same optimal frequency of 120 beats per min (bpm). This argument was predicated on the fact that participants adopt a spontaneous tempo of $120 \mathrm{bpm}$ when asked to finger tap (e.g., Kay, Kelso, Saltzman, \& Schoner, 1987) or walk (Hirasaki, Moore, Raphan, \& Cohen, 1999). Moreover, an analysis of over 70,000 pieces of popular music until 1990 indicated that $120 \mathrm{bpm}$ was the most prevalent tempo (MacDougall \& Moore, 2005). However, following a treadmill-based experiment, Schneider et al. reported that 2.7-2.8 Hz may represent the optimally efficient frequency for running at various intensities in line with the predictions of Gutmann, Jacobi, Butcher, and Bertram (2006). The music choices of participants to accompany the treadmill test appeared to fall in a similar range of 2.6-2.8 Hz (approximately 156-168 bpm). In criticism, the notion of optimal frequency does overlook the fact that this variable is specific to each individual and their intended work rate (cf. Smoll \& Schultz, 1982).

Hayakawa, Miki, Takada, and Tanaka (2000) assessed the effects of traditional Japanese folk music (used asynchronously) and the synchronous use of aerobics dance music on several dimensions of mood during a 30-min bench-stepping exercise. Both music conditions lessened feelings of fatigue when compared to the metronome control condition. Furthermore, aerobic dance music was responsible for increases in feelings of vigour when compared to a metronome control condition, whereas the folk music was not.

Also evaluating the psychological effects of synchronous music, van der Vlist, Bartneck, and Maüeler (2011) developed an interactive music feedback system akin to the Nike Plus interface. The system, named MoBeat, adjusts the musical tempi to synchronise with participants' cycling pace and issues recorded instructions to increase or reduce speed as appropriate. Furthermore, MoBeat adds additional instrumentation to the music track as a 'reward' for tempo compliance. Whereas the system did enhance participants' intrinsic motivation and attentional focus when compared to a heart rate (HR) monitor reference condition, no differences were evident in terms of perceived exertion and compliance to the prescribed movement tempo. Although at the vanguard of technological development in this field, the results may have been influenced by an elementary drawback. The synthesised music used was unfamiliar to participants and consequently had no motivational qualities by virtue of association. This is highly likely to have limited its impact. 
A number of studies found that synchronous music improved performance in motoric tasks (Karageorghis et al., 2009; Simpson \& Karageorghis, 2006; Terry, Karageorghis, Mecozzi Saha, \& D'Auria, 2012). In each case, the ergogenic effects of motivational music exceeded those of oudeterous (neutral) music. In the former study, music was used synchronously to ergogenic effect during a 400 -m sprinting task. The runners strode in time with a piece of motivational music that was delivered during the task. This music elicited faster times than a no-music control condition. A second synchronous condition consisting of music that was lacking in motivational qualities also improved performance relative to the control, but not to the same degree. Hence, both synchronisation and the motivational qualities of the music appear to be of benefit to high-intensity physical activity. Terry et al. also used a running task: a submaximal treadmill run to the point of volitional exhaustion. A sample of elite triathletes was able to endure longer in the presence of two synchronous music conditions (self-selected and an alternative that was neutral in motivational terms). Furthermore, mood responses and feeling states were more positive under the motivational music compared to either neutral or no-music conditions. Based on physiological indices, the authors contested that the synchronous music improved running economy by virtue of lower oxygen consumption and blood lactate levels.

Karageorghis et al. (2009) investigated the ergogenic effects of the synchronous application of music during treadmill walking. Participants began the task at $75 \%$ of their maximum heart rate and continued walking until voluntary volitional exhaustion. In addition to the no-music control, two synchronous conditions were employed: motivational and oudeterous music. Both music conditions promoted greater endurance than the control, with the motivational music proving superior to the oudeterous selection. Indeed, the motivational music elicited a marked $15 \%$ increase in treadmill endurance when compared to the control, and a clear 6\% increase relative to the oudeterous condition.

The experimental conditions appeared to lower perceptions of exertion at the start of the test relative to the remaining conditions. Nonetheless, as the intensity of exercise increased throughout the duration of the test, ratings of perceived exertion (RPE) ceased to differ by music condition. This trend marginally failed to achieve significance, but is in keeping with the prediction that the influence of music on attentional processes wanes at higher exercise intensities (cf. Rejeski, Tenenbaum). The Karageorghis et al. (2009) study also showed that music conditions exerted a strong influence on feeling states, which were more positive under the motivational condition until the point of volitional exhaustion. This result contradicts existing theory (Rejeski, 1985; Tenenbaum, 2001) given that the physiological strain associated with high-intensity exercise is expected to prevent participants from fully attending to musical stimuli. The findings supported the notion that, although music may not moderate what one feels during high-intensity exercise (i.e., RPE), it can moderate how one feels it (see Hardy \& Rejeski, 1989), therefore intense exercise can be more pleasurable with appropriate musical accompaniment. This result was mirrored by Shaulov and Lufi (2009) who investigated the effects of dance music when used synchronously during stationary cycling. While music did not lead to greater energy expenditure (calories burnt) when compared to a no-music control, participants did report heightened states of pleasure during the music condition. 
Styns, van Noorden, Moelants, and Leman (2007) conducted multiple trials to investigate the precise contingencies of the synchrony between walking and various music tempi (50-190 b pm). Whereas the overwhelming majority of participants chose to synchronise their movement to the musical tempo without solicitation, several anomalies were reported. On occasion participants synchronised to every half or every second beat. Also, the authors proposed that aspects of the music additional to tempo may influence the spatial aspects of movement. These aspects included the number of events per musical beat (meter), the number of musicians performing, and the energy with which instruments appeared to be played. Finally the authors proposed that any musical rhythm perpetuates an internalised 'model' of movement which may be generalised (not specific to listener) and is likely to consist of dance elements.

Hypothesising that the synchronous effects of music were more likely to become evident when motoric tasks of greater complexity were used, Karageorghis et al. (2010) assessed whether the performance of strength endurance exercises was affected by three synchronous conditions each delivered at a tempo of $120 \mathrm{bpm}$. Motivational music, oudeterous music and an audible metronome (control) were used to accompany sit-ups, squats, jumping jacks, heel raisers, step-ups and press behind neck. Each of these circuit-type exercises was performed until synchronous movement could no longer be maintained, and a gender comparison was a key feature of the study. The music conditions neither led to increases in the number of repetitions performed nor influenced feeling states when considering both genders together. However, a gender $\times$ condition interaction emerged: both genders performed worse when listening to oudeterous as opposed to motivational music, but whereas women performed similarly whether listening to oudeterous music or the control, men performed as well under the control condition as they had when listening to motivational music (see Figure 1a). Women also reported more positive feeling states than men in both music conditions (see Figure 1b). The men appeared

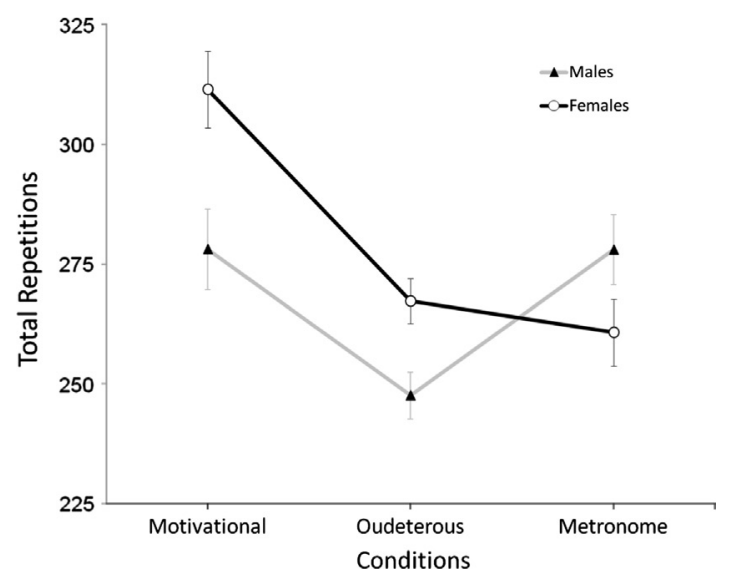

Figure 1a. Music condition $\times$ gender interaction for total repetitions $(p<.05)$. (Reprinted from Psychology of Sport and Exercise, 11, Karageorghis, C.I., Priest, D. L., Williams, L.S., Hirani, R.M., Lannon, K.M., \& Bates, B.J., Ergogenic and psychological effects of synchronous music during circuit-type exercise, 551-559, 2011, with permission from Elsevier.) 


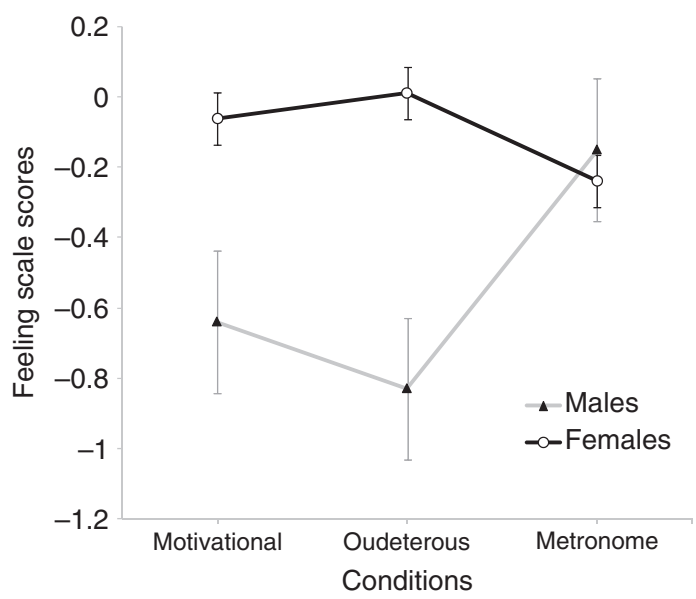

Figure $1 \mathrm{~b}$. Music condition $\times$ gender interaction for affect $(p<.05)$.

to find it harder than the women to coordinate their movements with musical tempo and this is perhaps reflected in the popularity of choreographed exercise classes among women. There is qualitative evidence to suggest that females experience a higher incidence of feeling 'motivated' by music than males (Priest, Karageorghis, \& Sharp, 2004). Age was an even stronger determinant of musical reactivity in the exercise setting, with older participants ( $>46$ years) starkly preferring less stimulative music, being less likely to report feeling 'motivated' by music, and regarding music during exercise as less important than their younger counterparts $(<26$ years $)$.

\section{Longitudinal effects of music}

Only two studies consider the longitudinal effects of music. Seath and Thow (1995) used pop music and an auditory metronome control condition (both 132 bpm) to accompany a four-week course of biweekly exercise sessions. The activity consisted of $15 \mathrm{~min}$ of aerobic exercise taking the form of a simplified exercise-tomusic dance routine. The music exerted large differences both on measures of RPE and positive feeling states, lowering the former and raising the latter. A minor criticism of this study is that RPE and affect measures were taken every 30 $\mathrm{s}$, a procedure that may have proved quite distracting/irritating for participants. Annesi (2001) reported that, over 14 weeks, members of a large private health club in the US experienced less dropout and trend towards greater attendance when exposed to an intervention that combined self-selected music with television (62 changeable channels) as opposed to interventions that comprised only music, only television, and neither of the above. Although clearly high in terms of ecological validity, the internal validity of this study is questionable on several grounds, chief among these being the small group sizes $(n=11-14)$ for independent sample analysis. Notably, the music-only condition fell marginally 
short of significance on several of the measures despite apparent meaningful differences (effect sizes were not reported).

Further corroboration of chronic effects that stem from listening to music during exercise can be found in the qualitative work based on semi-structured interviews (Priest \& Karageorghis, 2008) and nationwide surveys (Priest et al., 2004). In both studies respondents stated that music may positively (and in some cases negatively) influence their adherence to exercise and gym attendance.

\section{Post-task music}

The post-task use of music is typically recuperative in nature in that it aids recovery from injury, competition or training (Terry \& Karageorghis, 2011). At present, there has been very little empirical investigation into the capacity of music to aid recovery from training, competition or injury. However, music has been shown to offer pronounced recuperative effects in other areas such as education and health (e.g., DeNora, 2007; Särkämö et al., 2008; Sokhadze, Stewart, \& Hollifield, 2007). For example, Knight and Rickard (2001) reported that relaxing music prevents stress-induced increases in subjective anxiety, systolic blood pressure and heart rate. Sokhadze et al. have demonstrated that various types of music accelerated recovery from exposure to a stressful visual stimulus (images previously rated as 'disgusting'). Similarly, Hirokawa and Ohira (2003) found that listening to music after a stressful task led to measureable improvements in immune function, neuroendocrine responses and emotional states in college students. Finally, Labbé, Schmidt, Babin, and Pharr (2007) observed that listening to self-selected or classical music, after exposure to a stressor, significantly reduced the incidence of negative emotional states and physiological arousal when compared to listening to heavy metal music or sitting in silence.

Jing and Xudong (2008) have conducted what is possibly the inaugural study into the effects of post-task music in the exercise domain. A sedative instrumental piece (Acupuncture on the Mind composed by the second author) was used to aid the recovery of male college students for $15 \mathrm{~min}$ following an exhaustive cycle ergometer trial which began at $80-85 \%$ blood lactate threshold. Decreases in heart rate, urinary protein (indicative of post-exercise kidney function) and RPE were greater in the music group than the control. However, there was no difference in terms of the remaining measures - jump height, blood glucose, blood lactic acid and simple reaction time. This result indicates that post-exercise music use may have beneficial consequences although further studies to investigate the contingencies of its use are clearly required. The study, while groundbreaking, is not without its flaws. For example, a vertical jump is a questionable measure of fatigue while the 15-point Borg scale was certainly not designed for postexercise use.

Savitha, Mallikarjuna, and Chythra (2010) reported that slow music accelerated the haemodynamic (blood pressure and HR) recovery from $5 \mathrm{~min}$ of intense treadmill work when compared to fast music and a control. RPE decreased faster under the music condition in congruence with the physiological measures. Despite the fact that the study may have been flawed, for example no counterbalancing procedure appears to have been used, the results augment those of Jing and 
xudong (2008) and may be used to support the proposition that sedative music hastens post-exercise recovery. At this juncture, there is clearly scope for considerable additional work in the area of post-task music use.

\section{General discussion}

The purpose of this two-part review paper was to critically evaluate and synthesise research into the effects of music in an exercise context, with the aim of identifying future directions for research and providing evidence-based guidelines for practitioners. The principal delineator of the studies reviewed was the time of music's introduction relative to the task. It is evident that pre-task music can promote heightened arousal, both of the psychological and physiological variety. It is logical to infer that music can also act as a sedative when used pre-task, although there is currently insufficient empirical evidence in support of this; very few people need to be sedated or calmed before engaging in exercise. Those engaging in motoric activities that require elevated levels of psychomotor arousal are likely to prefer stimulative music (North \& Hargreaves, 2008). The efficacy of pre-task music use depends on how functional such changes in arousal/activation are for the listener and task in question. For example, an extravert would likely benefit from such an intervention prior to a bench press effort. Qualitative research (e.g., Priest \& Karageorghis, 2008) has begun to detail a more precise picture of the cognitions that accompany this 'psyching-up' process and, consequently, the paths that practitioners may use to activate individual athletes.

Of the 32 eligible studies in this review, 24 report an ergogenic effect when music is used in-task 27 refer to the asynchronous use of music and 23 report an ergogenic effect. The majority of studies that include a performance measure (e.g., distance travelled, repetitions completed) are focused on high-intensity exercise (e.g., tasks completed to the point of volitional exhaustion). Nevertheless, all six studies that focus on a low-to-moderate-intensity task and include a performance measure report that music promotes an ergogenic effect. Furthermore, during exercise of a low-tomoderate intensity, music is associated with a $\sim 10 \%$ reduction in RPE. This result is consistent across studies, even when the music is arbitrarily selected (e.g., classical music; Szmedra \& Bacharach, 1998) although studies that have manipulated music preference show that the largest benefits to RPE are associated with music that is preferred (e.g., Dyrland \& Wininger, 2008). There are also instances of participants performing greater work output with music while RPE remains stable or does not rise in the expected manner (e.g., Edworthy \& Waring, 2006; Elliott, Carr, \& Orme, 2005).

In essence, the key role of music in recreational exercise is one of lowering perceptions of exertion and thereby increasing the amount of work performed without the shift towards negative feeling states typically associated with more intense exercise (see Ekkekakis, Hall, \& Petruzzello, 2004; Hutchinson \& Tenenbaum, 2006). Typically, the psycho-physiological changes promoted by music are masked by those which accompany exercise. There are exceptions, however. For example, Urakawa and Yokoyama (2005) reported that listening to favoured musical selections during a 15 -min submaximal cycling bout led to an increased ratio 
of low- to high-frequency heart rate variability, which is indicative of sympathetic nervous system activity and hence increased arousal.

In total, 10 studies were concerned with the use of music during short-duration, high-intensity tasks; in all but two, music promoted ergogenic effects. When in-task music was used over a longer duration, during bouts of work completed to exhaustion, similar results ensued in that ergogenic effects were reported in 12 of the 14 studies cited. Although the internal feedback associated with exertion during high-intensity exercise dominates the perceptual field, music has the capacity to enhance positive feeling states during this type of exercise. Indeed, this improvement in affect appears to alter the way judgements are made about the exercise experience. Hence, the affective impact of music may be key to its positive influence on endurance during high-intensity exercise. This would not be illogical, as high-intensity exercise is associated with significant decrements in affect (see, e.g., Ekkekakis et al., 2004). Future research may be used to pinpoint whether the potency of music during higherintensity work is mediated by lyrical affirmations and task-related imagery.

Music, especially when selected according to its motivational qualities, renders moods and feeling states more positive across a range of exercise modalities and tasks (e.g., Karageorghis et al., 2009; Shaulov \& Lufi, 2009; Terry et al., 2012). This positive effect appears to hold regardless of exercise intensity or the synchronicity of the music, and ranges from $3-19 \%$ depending on how well the music is selected (i.e., with participants' preferences and task characteristics in mind). Well-selected music is associated with increases in positive affect that are greater than $10 \%$. The finding that music's affective qualities impact upon the psychological state of the exerciser when working at high intensities ( $>70 \%$ max heart rate reserve [HRR]) challenges the information processing model advanced by Rejeski (1985) who proposed that the internal feedback associated with physical exertion would predominate. However, convincing evidence has been revealed herein that, while music cannot alter what one feels when working at high intensities, it can still impact upon how one feels (Hardy \& Rejeski, 1989; Hutchinson et al., 2011; White \& Potteiger, 1996).

A number of authors (e.g., Annesi, 2001; Karageorghis, Terry, \& Lane, 1999; Miller, Swank, Manire, Robertson, \& Wheeler, 2010; Schwartz, Fernhall, \& Plowman, 1990) have proposed that the positive effects of music on feeling states can lead to increased adherence to exercise (i.e., attendance). This notion is also supported by interview-based evidence (DeNora, 2000, pp. 89-103; Priest \& Karageorghis, 2008). To elaborate on the distinction that Scherer (2004) drew between utilitarian and aesthetic emotions, it is probable that the aesthetic emotional response applies to low-to-moderate-intensity exercise whereas utilitarian emotions, which are adaptive and associated with pressing circumstances, are more relevant to high-intensity work.

While music that is selected according to its motivational qualities appears to have the greatest impact, it is clear that music per se leads to measurable benefits, both in terms of lowered RPE and an ergogenic effect. Accordingly, the Karageorghis et al. (1999) prediction that only motivational music is associated with reductions in perceived exertion has not been borne out by the 12 years of research that have followed. When music is used asynchronously, it appears to be at its most effective when used in conjunction with self-paced tasks, which mirror the ad hoc behaviour of exercise participants in vivo. Indeed, music use was associated with an ergogenic effect in each of the six studies where participants were permitted to select 
their own exercise intensity. Finally, music appears to be of greater benefit to untrained or recreationally active individuals than it does to those who are highly trained (e.g., Brownley, McMurray, \& Hackney, 1995; Mohammadzadeh, Tartibiyan, \& Ahmadi, 2008). This may be attributable to motivational differences between the two groups or the fact that, whereas trained individuals tend to work at high intensities, the untrained do not.

The observed ergogenic effect of music when used synchronously is concurrent with the documented predisposition of the human organism to respond physically to the rhythmical qualities of music (see, e.g., Crust, 2008; Karageorghis et al., 1999; Lucaccini \& Kreit, 1972). Various neuropsychological mechanisms have been advanced to explain this predisposition, including the presence of a neural pacemaker (Wilson, 1987, pp. 131-132), a property of the nervous system used to control repetitive movement known as timeform printing (Clynes \& Walker, 1982) and the dual involvement of certain brain sectors in music response and movement (Kornysheva, von Cramon, Jacobsen, \& Schubotz, 2010).

There is a weakness in approach that has limited research into the synchronous use of music. Very few studies have examined the relative effects of music used synchronously vs. asynchronously (e.g., Anshel \& Marisi, 1978). Hence, a detailed comparison between the effectiveness of these types of music use is not possible at the present time. Yet it is logical to infer that, when movements are synchronised to music, additional benefits are obtained both in terms of dissociation (through having to focus on keeping time) and energy efficiency in repetitive endurance activities and those with a marked rhythmic component (e.g., callisthenic-type exercises).

While relatively little is known about the impact of music heard post-task and used in a recuperative capacity, it is logical to infer from studies outside the sport and exercise domain that music has the capacity to relieve stress and improve affective states, thus aiding recovery (e.g., Särkämö et al., 2008; Sokhadze et al., 2007). This area is worthy of further systematic investigation as there are currently only two studies that the authors could locate, neither of which were particularly strong in methodological terms.

A prominent trend to emerge from the present review is that music appears to have a greater effect when external validity is relatively high, especially when either the task or music selection is self-regulated (as is often the case during exercise). This draws on self-determination theory insofar as autonomy is one of the three primary needs that underlie intrinsic motivation (Ryan \& Deci, 2000). In support of this point is the finding of Dwyer (1995) who found that post-exercise intrinsic motivation was higher in a group who were exposed to music they believed they had chosen, as opposed to a group who listened to the same music but were not informed that it was selected as a result of their surveyed preferences.

The effects of music with video in combination have received scant attention despite the fact that this type of delivery is rapidly usurping the former role of music, particularly in health and fitness facilities. In this sense, the experimental literature is entirely out of kilter with the real world. There is a dearth of work examining the motivational effects of moving images and whether the effects of music and video appose or interact in some manner. Both pieces of work featuring a music-video condition reported beneficial effects which bettered those of music alone (Annesi, 2001; Barwood, Weston, Thelwell, \& Page, 2009). Furthermore, Loizou and Karageorghis (2009) report that the use of primes incorporated into pre-task 
motivational video presentations led to performance increments in anaerobic work. The primes in this case were motivating words (e.g., 'push', 'drive', and 'go') which appeared momentarily (40 ms) on screen during the video presentations.

Finally, qualitative work has revealed many subtleties in musical response hitherto unreported in the experimental literature. For example, exercise participants have reported a strong anticipation effect whereby particular segments of musical pieces were awaited and used as a conscious trigger for increased work output (Priest \& Karageorghis, 2008). This effect was expressed in terms of heightened arousal during the bars leading up to the preferred segment. Such findings indicate a particularly nuanced and complex relationship between exerciser, task and music. Experimental work by Lim, Atkinson, Karageorghis, and Eubank (2009) using cycle ergometry with differentiated music exposure has also pointed towards similar anticipation effects, therefore this is a phenomenon that warrants further investigation.

\section{Directions for future research}

The present review bears several implications for future research through the trends and gap teeth in the literature that it has covered. Notably, the role of age, gender and other personal variables in musical reactivity within the exercise context is currently an underexplored area. For example, is it the case, as has been suggested in qualitative investigations (e.g., Priest \& Karageorghis, 2008), that music is a more powerful stimulus for younger exercise participants than it is for seniors? Perhaps the lack of research into age-congruent music has limited our understanding and so researchers should consider experimental protocols that entail age-congruent and age-incongruent music programmes.

As noted by Crust and Clough (2006), an important personal factor that has been largely overlooked in the literature is the mediating influence of personality type. An obvious entrée into this line of research would be to examine extraverted vs. introverted exercise participants with the hypothesis that extraverts would be likely to respond more favourably to loud/stimulative musical selections than introverts (cf. Eysenck, 1967). Similarly, attentional style and musical response in the exercise domain has received scant attention; it is thought that dissociators are likely to derive greater benefit from music use than associators, although an important mediating variable is task intensity. There is emerging evidence that cognitive strategies change in accordance with the intensity of an exercise task (Hutchinson \& Tenenbaum, 2006, 2007) which means that low-intensity tasks leave greater attentional capacity for parallel processing tasks such as music listening (cf. Tenenbaum, 2001). Hence, examining responses to music across a range of exercise intensities while employing participants who exhibit different attentional styles would be an especially propitious endeavour.

Researchers should also consider the extent to which music moderates ratings of perceived exertion beyond the anaerobic threshold, when physiological cues tend to predominate attentional processes. When a multi-dimensional approach is taken in the measurement of perceived exertion (e.g., Hutchinson \& Tenenbaum, 2006) it appears likely that carefully selected music (e.g., motivational music) can influence the motivational-affective component of perceived exertion beyond the anaerobic threshold, even if it cannot alter gestalt perceptions of exertion at such intensities. It 
is certainly the case that the degree to which the motivational qualities of music mediate reductions in perceived exertion warrants further empirical investigation.

Work to date has not systematically investigated the role of the lyrical content of music in determining psychological or psycho-physiological responses during exercise. It is plausible that the additional information processing capacity required to process lyrics can increase the dissociative influence of music, but there is a notable lack of exercise-related studies that compare responses to the same piece of music with and without lyrics. There is just one recent conference paper which showed that although lyrics contributed to the perceived motivational qualities of music, they had no bearing on psycho-physiological responses or work output in a submaximal cycle ergometer task (Sanchez, Moss, Twist, \& Karageorghis, 2011). Self-selected vs. experimenter-selected music has also been seldom tested (e.g., Dyrlund \& Wininger, 2008) although researchers should be aware of the inherent difficulties pertaining to self-selection; there is a greater likelihood for the emergence of Hawthorne effects and experimenter effects (see Lucaccini \& Kreit, 1972).

With reference to the cubic trend identified in the exercise heart rate-music tempo preference relationship (Karageorghis, Jones, et al., 2011), future researchers should seek to investigate the link between music tempo preference and a range of affective and motivational outcomes. This approach will reveal the psychological consequences of congruity between exercise heart rate and preferred music tempo. A replication of the Karageorghis, Jones, et al. study using other exercise modalities (e.g., cycling, motorised stepping, dance aerobics, etc.) and longer exercise bouts would shed light on the stability and applicability of the relationship. With reference to stability, it is unknown whether the cubic trend would manifest itself among middle aged and senior exercise participants, as it emerged through an investigation of young adults. Given that maximal heart rate gradually declines with age, one possibility is that the trend line will shift downwards and flatten when investigated among older groups of exercisers.

Research needs to be undertaken to evaluate the comparative effects of music used synchronously and asynchronously. In particular, do females derive greater benefit from the synchronous use of music when undertaking complex motor tasks such as callisthenic-type exercises or aerobic dance exercise (cf. Karageorghis et al., 2010)? Researchers may also consider the role of rhythmic ability in response to the synchronous use of music (cf. Roerdink, 2008, p. 13). Accordingly, there is scope to examine how syncopated (off beat) rhythms are processed by high and low rhythmic ability groups. There is a strong likelihood that individuals with high rhythmic ability and/or musical training will process such complex rhythms more easily, thus responding with greater bodily precision than their less musical counterparts (see, e.g., Vuust et al., 2005).

Research into post-task music use is in its infancy so there is considerable scope for work that ascertains how music facilitates recovery from injury and intense exercise. For this reason, it would be advantageous for researchers to develop and validate a tool, similar to the Brunel Music Rating Inventory (BMRI), for the assessment of the sedative qualities of music in an exercise context. In such investigations, an insightful approach would be the use of psychobiological measures, such as salivary cortisol, to examine the mechanisms that underlie the potential benefits of post-task music. 
The few studies that have examined training status as a moderator of exercisers' response to music have shown that this is an important personal variable that should be considered for inclusion in future research (Brownley et al., 1995; Mohammadzadeh et al., 2008). Two tentative findings that require more detailed investigation are that untrained participants tend to derive greater benefit from music, while those who are highly trained prefer to 'listen to their bodies' (i.e., use an associative attentional strategy). Nonetheless, the evidence presented herein indicates that, under certain circumstances (long-duration, low-intensity activities), highly trained individuals stand to benefit from the judicious use of music.

Work on the interactive effects of music and video is virgin territory for researchers, yet it represents a modality that is increasingly becoming ubiquitous in the gym environment (see Barwood et al., 2009; Loizou \& Karageorghis, 2009). Potential paths include the use of interview and observational techniques to gauge how exercisers respond to music and video across a range of exercise modalities.

The ultimate potential avenue for research is an investigation of the neurophysiological correlates of the musical response before, during and after exercise. The development of new technology, particularly $f$ MRI scanners that can be used during gross motor tasks, would facilitate such investigations. For example, this approach would be a possible means by which to explore the proposed 'attentional switching' (Rejeski, 1985) between internal and external cues that may take place at anaerobic threshold. A further possible exploration would entail evaluating the role of associations and memory in musical response and the shared activation between music and other emotive stimuli.

\section{Implications for exercise practitioners}

The evidence reviewed and synthesised in Parts I and II of this paper lead to a number of implications for exercise practitioners and participants. Music selected as an accompaniment for exercise should be congruent with participants' personal characteristics (e.g., age and socio-cultural upbringing), the exercise environment and desired outcomes. When working with a mixed group in terms of age and/or sociocultural upbringing, practitioners might consider democratising the music selection process so that all participants' musical predilections are accounted for and reflected in the music programme (Dyrlund \& Wininger, 2008; Nakamura et al., 2010).

Music tempo should be selected with the expected exercise intensity in mind, and be sequenced to contour in accordance with changes in heart rate. Other than when warming up, warming down or recuperating, the appropriate band of tempi for exercise intensities in the range 40-90\% max HRR is $125-140 \mathrm{bpm}$ when the music is used asynchronously (see Karageorghis, Jones, et al., 2011). It is also advantageous for the rhythm of the music to approximate the motor patterns entailed where possible (Crust, 2008; Schneider et al., 2010). In group exercise classes when music is used synchronously, it appears that the rhythmic abilities of participants warrant careful consideration in the sequencing of music selections (see, e.g., DeNora, 2000, pp. 8991). In self-directed exercise, synchronous motor activity may occur in association with complex rhythmical segments that involve syncopation or span musical measures, not simply with the principal beat that marks musical tempo (Styns et al., 2007).

Music containing affirmations of exercise or inspirational references drawn from popular culture should be selected in order to promote motivational imagery 
and self-talk. Positive affect is thought to be consequent to the harmonic and melodic features of the music and its lyrical content (Karageorghis et al., 1999; Lucaccini \& Kreit, 1972). In order to have a stimulative effect, the music should be up-tempo $(>120 \mathrm{bpm})$ and possess prominent percussive and rhythmical features. In order to sedate, the inverse is true in terms of percussion and rhythmical features, and tempi $<80 \mathrm{bpm}$ are recommended (see Terry \& Karageorghis, 2011).

A novel finding that has emanated from qualitative work is that music selection for exercise is concerned with the entire programme of music, not just individual pieces (e.g., DeNora, 2000, pp. 89-103; Priest \& Karageorghis, 2008), a perspective not countenanced in the experimental literature. For example, selectors should consider the congruence of musical pieces that appear in close proximity on a playlist and aim to achieve variety in terms of churn (i.e., varying selections from session to session). With specific reference to congruence, taking into account factors such as beat matching, style matching, artist matching, era matching, etc. is important in terms of formulating a cohesive music mix. Moreover, a change or absence of music can be an effective marker of the next exercise phase or unit (DeNora), such as the progression from a cardiovascular segment to a warm-down phase.

The findings reported in this review lead us to the conclusion that self-selected music is especially efficacious in an applied context (e.g., Bharani, Sahu, \& Mathew, 1984; Priest \& Karageorghis, 2008). It is exceedingly unlikely that a 'motivational' music programme selected by a practitioner would yield stronger effects than a programme selected by the participant themselves, unless they make inept choices. Self-selection also invokes a sense of autonomy, which is a central tenet of intrinsically motivated behaviour (Ryan \& Deci, 2000). Bearing in mind the individual variation in the desired psychological state during exercise and the subjective nature of music preference, there is a clear basis for encouraging the selfselection or democratisation of music selection where possible.

The role of exercise practitioners should be limited to guiding and focusing the selection process by helping individuals to identify motivational music and develop track selections into coherent programmes that contour the mental and physical demands of their workout (see, e.g., Karageorghis, Jones, et al., 2011). The use of objective music selection procedures (e.g., BMRI-2) would seem to be most apposite in the case of large groups of exercise participants, especially those that fluctuate over time such as the usership of a gym. In these cases, individual preferences and needs of the few are subsumed into a collective response (of the many). This is especially so when such groups are mixed in terms of gender, age and socio-cultural background.

Special attention should be given to the distinction between pre-, in-, and posttask music selection. We contend that at present practitioners give insufficient consideration to pre- and post-task music. Pre-task music can be used as an effective pre-exercise prime while post-task music can aid mental and physical revitalisation. While there should be a clear demarcation among the various applications, it is possible to maintain coherence in a music programme by using pieces from the same era, the same genre, or by the same artist when moving between the pre-, in-, and post-task phases.

Terry and Karageorghis (2011) recently proposed guidelines to aid the selection of post-task music. It should possess sedative qualities, such as a tempo in the range 60-70 bpm (around resting heart rate), a simplistic rhythmical 
structure, regular pulsation and repetitive tonal patterns based on a limited number of pitch levels. In terms of emotional tone, the music should be neutral or relaxing, while the instrumentation might be comprised of soothing, 'warm' instruments, such as strings, oboe or gentle piano. The inclusion of natural sounds, such as breaking waves, bird song or a babbling brook, may also be effective for this purpose.

According to Karageorghis, Terry, Lane, Bishop, and Priest (2011), while music is a beneficial accompaniment to exercise in most circumstances, it is contraindicated under certain conditions: (a) when it may distract users from safety-relevant information (e.g., on public roads); (b) when exercisers need to focus their full attention on learning a demanding motor skill (e.g., a power clean); and (c) when exercising at high intensities that require an associative attentional style (i.e., 'listening to the body'). It is probable that, as a stimulant, music should be used intermittently (i.e., not all of the time). This approach will prevent desensitisation to its stimulative effects and permit exercisers to habituate themselves for occasions when they may not be able to use it (e.g., long-distance running events in which music is not permitted). Excessively loud music combined with high-intensity exercise is to be avoided owing to the potential threat to the structures of the inner ear. Research shows that even moderate-intensity activity accompanied by very loud music ( $>100 \mathrm{~dB}$ sound pressure level) can cause temporary hearing loss (Lindgren \& Axelsson, 1988).

\section{Conclusions}

The evidence in this field demonstrates that music has the capacity to exert beneficial psychological and ergogenic effects across a wide range of exercise tasks and through a number of applications, pre-task, in-task and post-task. The evidence is not unilateral and there are certain circumstances, tasks and participant groups that are more conducive to these benefits than others. Nevertheless, with the exception of the psycho-physiological responses to music, the effects described herein are of a consistent nature. Music has a definitive, functional role in the exercise environment. It is notable that the findings which support these conclusions are influenced heavily by the heightened methodological scrutiny and theoretical framework that emerged in the late 1990s. From this point, researchers have generally adopted a more judicious approach to music selection and become more sensitive to participants' musical preferences and socio-cultural background. Researchers are now more aware of the inherent subjectivity of the stimulus under investigation and the complexity of its effects. Music is now rarely viewed in a manner akin to the 'vitamin model' described by Sloboda (2008, p. 32) wherein one can ascribe immutable effects to a specific musical selection for all listeners and at all times. The beneficial consequences of music use stem from an interaction between elements of the musical stimulus itself and factors relating to the traits and experiences of the listener, and aspects of the exercise environment and task. In particular, the role of music is dependent on when it is introduced in relation to the task and the intensity of the exercise undertaken. In closing, the evidence presented in this review demonstrates that music has a consistent and measurable effect on the psychological state and behaviour of exercise participants. 


\section{References}

Annesi, J.J. (2001). Effects of music, television, and a combination entertainment system on distraction, exercise adherence, and physical output in adults. Canadian Journal of Behavioural Science, 33, 193-201.

Anshel, M.H., \& Marisi, D.Q. (1978). Effects of music and rhythm on physical performance. Research Quarterly, 49, 109-113.

Barwood, M.J., Weston, J.V., Thelwell, R., \& Page, J. (2009). A motivational music and video intervention improves high-intensity exercise performance. Journal of Sports Science and Medicine, 8, 435-442.

Bharani, A., Sahu, A., \& Mathew, V. (2004). Effect of passive distraction on treadmill exercise test performance in healthy males using music. International Journal of Cardiology, 97, 305-306.

Brownley, K.A., McMurray, R.G., \& Hackney, A.C. (1995). Effects of music on physiological and affective response to graded treadmill exercise in trained and untrained runners. International Journal of Psychophysiology, 19, 193-201.

Clynes, M., \& Walker, J. (1982). Neurobiologic functions of rhythm time and pulse in music. In M. Clynes (Ed.), Music, mind and brain: The neuropsychology of music (pp. 171-216). New York: Plenum Press.

Crust, L. (2008). The perceived importance of components of asynchronous music in circuit training exercise. Journal of Sports Sciences, 23, 1-9.

Crust, L., \& Clough, P.J. (2006). The influence of rhythm and personality in the endurance response to motivational asynchronous music. Journal of Sports Sciences, 24, 187-195.

DeNora, T. (2000). Music in everyday life. Cambridge, UK: Cambridge University Press.

DeNora, T. (2007). Evidence and effectiveness in music therapy: Problems, possibilities and performance in health contexts. British Journal of Music Therapy, 20, 81-99.

Dwyer, J.J.M. (1995). Effect of perceived choice of music on exercise intrinsic motivation. Health Values, 19, 18-26.

Dyrlund, A.K., \& Wininger, S.R. (2008). The effects of music preference and exercise intensity on psychological variables. Journal of Music Therapy, 45, 114-134.

Edworthy, J., \& Waring, H. (2006). The effects of music tempo and loudness level on treadmill exercise. Ergonomics, 49, 1597-1610.

Ekkekakis, P., Hall, E E., \& Petruzzello, S.J. (2004). Practical markers of the transition from aerobic to anaerobic metabolism during exercise: Rationale and a case for affect-based exercise prescription. Preventive Medicine, 38, 149-159.

Elliott, D., Carr, S., \& Orme, D. (2005). The effect of motivational music on sub-maximal exercise. European Journal of Sport Science, 5, 97-106.

Eysenck, H.J. (1967). The biological basis of personality. Springfield, IL: Thomas Publishing.

Gutmann, A.K., Jacobi, B., Butcher, M.T., \& Bertram, J.E.A. (2006). Constrained optimization in human running. Journal of Experimental Biology, 209, 622-632.

Hardy, C.J., \& Rejeski, W.J. (1989). Not what, but how one feels: The measurement of affect during exercise. Journal of Sport \& Exercise Psychology, 11, 304-317.

Hayakawa, Y., Miki, H., Takada, K., \& Tanaka, K. (2000). Effects of music on mood during bench stepping exercise. Perceptual and Motor Skills, 90, 307-314.

Hirasaki, E., Moore, S.T., Raphan, T., \& Cohen, B. (1999). Effects of walking velocity on vertical head and body movements during locomotion. Experimental Brain Research, 127, $117-130$.

Hirokawa, E., \& Ohira, H. (2003). The effects of music listening after a stressful task on immune functions, neuroendocrine responses and emotional states of college students. Journal of Music Therapy, 60, 189-211.

Hutchinson, J.C., Sherman, T., Davis, L.K., Cawthon, D., Reeder, N.B., \& Tenenbaum, G. (2011). The influence of asynchronous motivational music on a supramaximal exercise bout. International Journal of Sport Psychology, 42, 135-148.

Hutchinson, J.C., \& Tenenbaum, G. (2006). Perceived effort - can it be considered gestalt? Psychology of Sport and Exercise, 7, 463-476.

Hutchinson, J.C., \& Tenenbaum, G. (2007). Attention focus during physical effort: The mediating role of task intensity. Psychology of Sport and Exercise, 8, 233-245. 
Jing, L., \& Xudong, W. (2008). Evaluation on the effects of relaxing music on the recovery from aerobic exercise-induced fatigue. Journal of Sports Medicine and Physical Fitness, 48, $102-106$.

Karageorghis, C.I., Jones, L., Priest, D.L., Akers, R.I., Clarke, A., Perry, J., ... Lim, H.B.T. (2011). Revisiting the exercise heart rate-music tempo preference relationship. Research Quarterly for Exercise and Sport, 82, 274-284.

Karageorghis, C.I., Mouzourides, D., Priest, D.L., Sasso, T., Morrish, D., \& Whalley, C. (2009). Psychophysical and ergogenic effects of synchronous music during treadmill walking. Journal of Sport \& Exercise Psychology, 31, 18-36.

Karageorghis, C.I., Priest, D.L., Williams, L.S., Hirani, R.M., Lannon, K.M., \& Bates, B.J. (2010). Ergogenic and psychological effects of synchronous music during circuit-type exercise. Psychology of Sport and Exercise, 11, 551-559.

Karageorghis, C.I., \& Terry, P.C. (1997). The psychophysical effects of music in sport and exercise: A review. Journal of Sport Behavior, 20, 54-68.

Karageorghis, C.I., Terry, P.C., \& Lane, A.M. (1999). Development and initial validation of an instrument to assess the motivational qualities of music in exercise and sport: The Brunel Music Rating Inventory. Journal of Sports Sciences, 17, 713-724.

Karageorghis, C.I., Terry, P.C., Lane, A.M., Bishop, D.T., \& Priest, D.L. (2011). British Association of Sport and Exercise Sciences expert statement on the use of music in exercise. The Sport and Exercise Scientist, 28, 18-19.

Kay, B.A., Kelso, J.A., Saltzman, E.L., \& Schoner, G. (1987). Space-time behavior of single and bimanual rhythmical movements: Data and limit cycle model. Journal of Experimental Psychology: Human Perception and Performance, 13, 178-192.

Knight, W.E.J., \& Rickard, N.S. (2001). Relaxing music prevents stress-induced increases in subjective anxiety, systolic blood pressure and heart rate in healthy males and females. Journal of Music Therapy, 38, 254-272.

Kornysheva, K., von Cramon, D.Y., Jacobsen, T., \& Schubotz, R.I. (2010). Tuning-in to the beat: Aesthetic appreciation of musical rhythms correlates with a premotor activity boost. Human Brain Mapping, 31, 48-64.

Labbé, E., Schmidt, N., Babin, J., \& Pharr, M. (2007). Coping with stress: The effectiveness of different types of music. Applied Psychophysiology and Biofeedback, 32, 163-168.

Lim, H.B.T., Atkinson, G., Karageorghis, C.I., \& Eubank, M. (2009). Effects of differentiated music exposure during a 10-km cycling time trial. International Journal of Sports Medicine, $30,435-442$.

Lindgren, F., \& Axelsson, A. (1988). The influence of physical exercise on susceptibility to noise-induced temporary threshold shift. Scandinavian Audiology, 17, 11-17.

Lucaccini, L.F., \& Kreit, L.H. (1972). Music. In W.P. Morgan (Ed.), Ergogenic aids and muscular performance (pp. 240-245). New York: Academic Press.

Loizou, G., \& Karageorghis, C.I. (2009). Video, priming and music: Effects on emotions and motivation. In A.J. Bateman \& J.R. Bale (Eds.), Sporting sounds: Relationships between sport and music (pp. 37-58). London: Routledge.

MacDougall, H.G., \& Moore, S.T. (2005). Marching to the beat of the same drummer: The spontaneous tempo of human locomotion. Journal of Applied Physiology, 99, $1164-1173$.

Miller, T., Swank, A.M., Manire, J.T., Robertson, R.J., \& Wheeler, B. (2010). Effect of music and dialog on perception of exertion, enjoyment, and metabolic responses during exercise. International Journal of Fitness, 6, 45-52.

Mohammadzadeh, H., Tartibiyan, B., \& Ahmadi, A. (2008). The effects of music on the perceived exertion rate and performance of trained and untrained individuals during progressive exercise. Facta Universitatis: Series Physical Education \& Sport, 6, $67-74$.

Nakamura, P.M., Pereira, G., Papini, C.B., Nakamura, F.Y., \& Kokubun, E. (2010). Effects of preferred and nonpreferred music on continuous cycling exercise performance. Perceptual and Motor Skills, 110, 257-264.

North, A.C., \& Hargreaves, D.J. (2008). Music and taste. In A.C. North \& D.J. Hargreaves (Eds.), The social and applied psychology of music (pp. 75-142). Oxford, UK: Oxford University Press. 
Priest, D.L., \& Karageorghis, C.I. (2008). A qualitative investigation into the characteristics and effects of music accompanying exercise. European Physical Education Review, 14, 347-366.

Priest, D.L., Karageorghis, C.I., \& Sharp, N.C.C. (2004). The characteristics and effects of motivational music in exercise settings: The possible influence of gender, age, frequency of attendance, and time of attendance. Journal of Sports Medicine and Physical Fitness, 44, 77-86.

Rejeski, W.J. (1985). Perceived exertion: An active or passive process? Journal of Sport Psychology, 75, 371-378.

Roerdink, M. (2008). Anchoring: Moving from theory to therapy. Amsterdam: IFKB.

Ryan, R.M., \& Deci, E.L. (2000). Self-determination theory and the facilitation of intrinsic motivation, social development, and well-being. American Psychologist, 55, 68-78.

Sanchez, X., Moss, S.L., Twist, C., \& Karageorghis, C.I. (2011). Role of lyrics in the musicperformance relationship: A preliminary study. In Proceedings of the 2011 FEPSAC Congress, Madeira, Portugal.

Särkämö, T., Tervaniemi, M., Laitinen, S., Forsblom, A., Soinila, S., Mikkonen, M., ... Hietanen, M. (2008). Music listening enhances cognitive recovery and mood after middle cerebral artery stroke. Brain, 131, 866-876.

Savitha, D., Mallikarjuna, R.N., \& Chythra, R. (2010). Effect of different musical tempo on post-exercise recovery in young adults. Indian Journal of Physiology and Pharmacology, 54, $32-36$.

Scherer, K.R. (2004). Which emotions can be induced by music? What are the underlying mechanisms? And how can we measure them? Journal of New Music Research, 33, 239-251.

Schneider, S., Askew, C.D., Abel, T., \& Strüder, H.K. (2010). Exercise, music, and the brain: Is there a central pattern generator? Journal of Sports Sciences, 28, 1337-1343.

Schwartz, S.E., Fernhall, B., \& Plowman, S.A. (1990). Effects of music on exercise performance. Journal of Cardiopulmonary Rehabilitation, 10, 312-316.

Seath, L., \& Thow, M. (1995). The effect of music on the perception of effort and mood during aerobic type exercise. Physiotherapy, 81, 592-596.

Shaulov, N., \& Lufi, D. (2009). Music and light during indoor cycling. Perceptual and Motor Skills, 108, 597-607.

Simpson, S.D., \& Karageorghis, C.I. (2006). The effects of synchronous music on 400-m sprint performance. Journal of Sports Sciences, 24, 1095-1102.

Sloboda, J. (2008). The ear of the beholder. Nature, 454, 32-33.

Smoll, F.L., \& Schultz, R.W. (1982). Accuracy of motor behaviour in response to preferred and nonpreferred tempos. Journal of Human Movement Studies, 8, 123-138.

Sokhadze, T., Stewart, C.M., \& Hollifield, M. (2007). Integrating cognitive neuroscience research and cognitive behavioral treatment with neurofeedback therapy in drug addiction comorbid with Posttraumatic Stress Disorder: A conceptual review. Journal of Neurotherapy, 11, 13-44.

Styns, F., van Noorden, L., Moelants, D., \& Leman, M. (2007). Walking on music. Human Movement Science, 26, 769-785.

Szmedra, L., \& Bacharach, D.W. (1998). Effect of music on perceived exertion, plasma lactate, norepinephrine and cardiovascular hemodynamics during treadmill running. International Journal of Sports Medicine, 19, 32-37.

Tenenbaum, G. (2001). A social-cognitive perspective of perceived exertion and exertion tolerance. In R.N. Singer, H.A. Hausenblas, \& C. Janelle (Eds.), Handbook of sport psychology (pp. 810-822). New York: Wiley.

Terry, P.C., \& Karageorghis, C.I. (2011). Music in sport and exercise. In T. Morris \& P.C. Terry (Eds.), The new sport and exercise psychology companion (pp. 359-380). Morgantown, WV: Fitness Information Technology.

Terry, P.C., Karageorghis, C.I., Mecozzi Saha, A., \& D’Auria, S. (2012). Effects of synchronous music on treadmill running among elite triathletes. Journal of Science and Medicine in Sport. doi: 10.1016/j.jsams.2011.06.003

Urakawa, K., \& Yokoyama, K. (2005). Music can enhance exercise-induced sympathetic dominancy assessed by heart rate variability. Tohoku Journal of Experimental Medicine, 206, 213-218. 
van der Vlist, B., Bartneck, C., \& Mäueler, S. (2011). moBeat: Using interactive music to guide and motivate users during aerobic exercising. Applied Psychophysiology and Biofeedback, 36, $135-145$.

Vuust, P., Pallensen, K.J., Bailey, C., van Zuijen, T.L., Gjedde, A., Roepstorff, A., \& Østergaard, L. (2005). To musicians, the message is in the meter. Pre-attentive neuronal responses to incongruent rhythm are left-lateralized in musicians. NeuroImage, 24, 560-564. Wilson, F.R. (1987). Tone deaf and all thumbs?. New York: Vintage.

White, V. B., \& Potteiger, J. A. (1996). Comparison of passive sensory stimulations on RPE during moderate intensity exercise. Perceptual and Motor Skills, 82, 819-825. 